\title{
COMPARAÇÃO DE PARTÍCULAS TOTAIS EM SUSPENSÃO VIA DETERMINAÇÃO GRAVIMÉTRICA COM AMOSTRAGEM DE GRANDE E PEQUENO VOLUME NO ESTADO DO PARANÁ, BRASIL COMPARISON OF TOTAL SUSPENDED PARTICLES MEASUREMENTS VIA HIGH AND LOW VOLUME SAMPLING IN THE STATE OF PARANA, BRAZIL
}

\section{Paulo Eduardo Slapnig}

Mestre em Gestão Ambiental pela Universidade Positivo (UP). Ex-aluno do Programa de Pós-Graduação em Gestão Ambiental da UP Curitiba (PR), Brasil.

\section{Eliane Carvalho Vasconcelos (iD}

Doutora em Ciências pela Universidade de São Paulo (USP) São Carlos, SP. Professora no Programa de Pós-Graduação em Gestão Ambiental e Mestrado em Biotecnologia Industrial da UP Curitiba (PR), Brasil.

\section{Paulo Roberto Janissek}

Doutor em Química Orgânica pela USP, Professor no Instituto Federal de Educação, Ciência e Tecnologia do Rio Grande do Sul, Campus Caxias do Sul-Caxias do Sul (RS), Brasil.

\section{Endereço para correspondência:} Eliane Carvalho Vasconcelos Rua Professor Pedro Viriato Parigot de Souza, 5.300 - Campo Comprido - CEP 81280-330 Curitiba (PR), Brasil - E-mail: evasconcelos@up.edu.br

Recebido em: 18/08/2016

Aceito em: 19/11/2018

\section{RESUMO}

Foram realizadas determinações de partículas totais em suspensão aplicandose em paralelo dois métodos gravimétricos distintos: da amostragem de grande volume, conforme metodologia de referência estabelecida na Associação Brasileira de Normas Técnicas (ABNT), Norma Brasileira (NBR) no 9.547/97; e da amostragem de pequeno volume, conforme método definido neste estudo. As amostragens ocorreram de outubro de 2011 a janeiro de 2012, no estado do Paraná, Brasil, em locais com características distintas. O primeiro local em área urbana, sem atividades industriais vizinhas; no segundo local predominava em seu entorno uma indústria papeleira; e no terceiro, a atividade de armazenamento e distribuição de fertilizantes. O método do amostrador de pequeno volume apresentou resultados equivalentes ao método de referência na faixa de 0 a $100 \mu \mathrm{g} \cdot \mathrm{m}^{-3} \mathrm{em}$ ambientes com particulado fino. Verificaram-se resultados discrepantes em ambiente com partículas de diâmetros maiores. Esses resultados foram atribuídos à limitação do ajuste inicial de vazão com o método de referência e sua limitação de controle ao longo da amostragem. A ampla faixa de velocidade de aspiração de amostra admitida para o método de referência aumenta a incerteza de seus resultados, principalmente em atmosferas com partículas de maior diâmetro. O método da amostragem de pequeno volume proposto demonstrou ser uma alternativa mais moderna e vantajosa para determinação de partículas totais em suspensão, se comparado ao método de referência, e possibilita maior flexibilidade e menor incerteza de resultados decorrentes da amostragem.

Palavras-chave: poluição atmosférica; qualidade do ar; métodos; particulado total em suspensão; partículas totais em suspensão; ABNT NBR 9.547; amostrador de grande volume; amostrador de pequeno volume.

\section{ABSTRACT}

Total suspended particles determinations were performed in parallel with two different gravimetric methods: the high-volume sampling method, reference methodology established in Brazil by ABNT NBR 9,547/97, and the low-volume sampling method, defined in this study. Samples were undertaken from October/2011 to January/2012, in the state of Paraná, Brazil, at three locations with different human activities. The first was mainly urban, with no industries around. The second had a paper mill in the surroundings and the third had around fertilizer handling, transportation and storage. Low-volume sampling method generated equivalent results to the reference method at the range 0 to $100 \mu \mathrm{g} \cdot \mathrm{m}^{-3}$ in atmospheres with fine particulate. The atmosphere with greater particles generated discrepant results. This was due to the reference sampler limitations atinitial flow adjustment and flow control during sampling period. The wide range admitted to the sampling air velocity at the reference method increases the uncertainty of its results, mainly in atmospheres with 
greater particles. The low-volume method proposed at this study is a more advanced alternative with advantages for total suspended particles measurement if compared to the reference method,and it is a more flexible alternative method that carries less uncertainty of results assigned to sampling.

Keywords: atmospheric pollution; air quality; methods; total suspended particles; ABNT NBR 9,547; high volume sampling; low volume sampling.

\section{INTRODUÇÃO}

As evidências epidemiológicas verificadas nas populações, conforme estudos conduzidos na Europa e na América, demonstraram efeitos adversos à saúde de indivíduos pela exposição a partículas em suspensão por longo e curto período (WHO, 2005). Diversos pesquisadores têm avaliado os efeitos da concentração de partículas no ar sobre alguns aspectos da saúde humana. Estudos verificaram que para cada incremento de $10 \mu \mathrm{g} \cdot \mathrm{m}^{-3}$ de particulado com menos de $10 \mu \mathrm{m}$ de diâmetro aerodinâmico (PM10) houve excesso de internações de 4,25\% para doenças respiratórias totais, de $5,74 \%$ para doenças respiratórias em menores de 5 anos, e de 2,29\% para doenças cardiovasculares em maiores de 39 anos (NARDOCCl et al., 2013). A exposição ao material particulado com menos de $2,5 \mu \mathrm{m}$ de diâmetro aerodinâmico (PM2.5) esteve associada às internações por doenças respiratórias em crianças (CESAR; NASCIMENTO; CARVALHO, 2013). Praticantes de exercícios em locais com elevadas concentrações de material particulado podem ter redução de suas funções pulmonares (KESAVACHANDRAN et al., 2015). Os efeitos adversos da exposição ao particulado inalável vão além das doenças cardiorrespiratórias, pois aumentam o risco de recém-nascidos com baixo peso (ROMÃO et al., 2013). E há inclusive estudos que relatam associação entre o nível de PM10 e a mortalidade (BLANCO-BECERRA et al., 2014; CESARONI et al., 2013). Entretanto, não se sabe precisar atualmente sobre o nível de exposição ao particulado que não causa efeitos nocivos ao ser humano (KIM; KABIR; KABIR, 2015; KUMAR; ATTRI, 2016).

As chamadas partículas inaláveis, ou PM10, são a fração das partículas totais em suspensão (PTS) com maior potencial para causar danos à saúde, motivo pelo qual a Agência de Proteção Ambiental Americana substituiu o PTS pelo PM10 na definição do padrão nacional de qualidade do ar, em 1987 (DAUMAS; MENDONÇA; LEON, 2004). Na comunidade Europeia, a fração de interesse e alvo de definição de limite legal de concentração também é o PM10, e não há padrão para PTS, segundo o Comitê Técnico da Comunidade Europeia (ECTC, 2010).

Entretanto, alguns países ainda mantêm padrões para PTS, tais como Brasil, Colômbia e México (BRASIL, 1990; COLÔMBIA, 1982; MÉXICO, 2005). No Brasil, os padrões de qualidade do ar para PM10 estão estabelecidos em $50 \mu \mathrm{g} \cdot \mathrm{m}^{-3}$ para média aritmética anual, e em $150 \mu \mathrm{g} \cdot \mathrm{m}^{-3}$ para média de 24 horas. E os padrões para PTS estão estabelecidos em $80 \mu \mathrm{g} \cdot \mathrm{m}^{-3}$ para média anual e em $240 \mu \mathrm{g} . \mathrm{m}^{-3}$ para média de 24 horas (BRASIL, 1990).

A determinação da concentração de partículas não é simples, pois existe uma variedade de técnicas de medição, e por conta da complexidade da natureza do material particulado, a escolha do método de medição pode influenciar no resultado. Alguns métodos fornecem resultados contínuos, tais como analisadores por $\beta$ atenuação, analisadores óticos, monitores tipo microbalança oscilante/elemento cônico, e outros são gravimétricos, dependem da coleta em filtros e de pesagem do material coletado (REINO UNIDO, 2005).

Nos Estados Unidos, conforme a Agência de Proteção Ambiental Americana (USEPA, 2011), o método de referência e aprovado para determinação de PTS está estabelecido em 40 CFR, Part 50, Apendix B, de 1982, via amostragem de grande volume (AGV) e gravimetria. Segundo a Associação Brasileira de Normas Técnicas (ABNT, 1997), no Brasil, a metodologia de referência e aprovada para a determinação de PTS é a ABNT/Norma Brasileira (NBR) $n^{\circ}$ 9.547/97, via AGV, elaborada com 
base na norma americana. Outros países da América Latina também tiveram seus métodos de referência para determinação do PTS com base na norma americana, tais como México e Colômbia (MÉXICO, 2005; COLÔMBIA, 1982).

No Brasil, os resultados de PTS obtidos com outros métodos podem ser considerados válidos, desde que equivalentes aos obtidos com o método de referência (BRASIL, 1990). Ou seja, a utilização de outros métodos requer de comprovação de sua equivalência de resultados. Diante dessa abertura legal e da alternativa de métodos mais modernos e vantajosos, porém localmente ainda não consagrados, este trabalho avaliou resultados de PTS obtidos com dois métodos gravimétricos distintos, o de referência e o da amostragem de pequeno volume (APV) definido neste estudo.

A determinação do PTS via método de referência, também chamado de AGV, consiste na aspiração de ar ambiente por meio de um filtro, abrigado, em que por meio das condições de vazão e dimensionamento da abertura de adução do ar estabelecidas no método, ocorre a captação de partículas de diâmetro até $50 \mu \mathrm{m}$ e em uma faixa de velocidade aproximada entre 20 e $35 \mathrm{~cm} . \mathrm{s}^{-1}$, correspondente à vazão de amostragem entre 1,1 e 1,7 $\mathrm{m}^{3} \cdot \mathrm{min}^{-1}$. Nesse método, a vazão e a velocidade de amostragem não têm controle automatizado ao longo da amostragem, mas somente ajuste inicial aproximado a partir do ajuste de um variador de tensão de alimentação do motor. $O$ ajuste inicial é aproximado, pois a vazão é verificada a partir do registro de deflexão em carta gráfica e cálculo subsequente, considerando-se temperatura e pressão atmosféricas, e os coeficientes de calibração a2 e b2. O aumento da perda de carga no filtro decorrente da massa de partículas acumulada, que de fato ocorre, e oscilações de energia ao longo da amostragem, também comuns, afetam diretamente a velocidade de sucção de amostra. Os filtros são acondicionados em umidade inferior a $50 \%$ por pelo menos 24 horas, até seu equilíbrio, e pesados com precisão de $0,1 \mathrm{mg}$, anteriormente e posteriormente à amostragem. A massa de partículas coletadas é obtida pela diferença do peso pós-amostragem e pré-amostragem. O peso obtido é utilizado para determinação da concentração de PTS a partir de sua divisão pelo volume amostrado em condições padrão. Este é obtido a partir do tempo de amostragem, da vazão de amostragem, da pressão atmosférica e da temperatura do ar. As principais partes do sistema de amostragem são casinhola de abrigo, motoaspirador, dispositivo de ajuste de vazão, dispositivo indicador/registrador de deflexão, dispositivo de programação de tempo, termômetro, barômetro e filtros de dimensões 20,3 $\times 25,4 \mathrm{~cm}$. É definido o uso de um calibrador padrão de vazão que pode ser de orifício com placas fixas, de orifício com resistência ajustável externamente ou de fluxo eletrônico com resistência ajustável externamente (ABNT, 1997).

O método para determinação de PTS via APV utilizou sistema de amostragem mais moderno e flexível, pois possui ajuste preciso da vazão e seu controle ao longo da amostragem. Além disso, permite a amostragem de PM10 e PM2.5 pela simples troca da cabeça de amostragem, de baixo custo. Utiliza filtros circulares com diâmetro de $47 \mathrm{~mm}$, acondicionados em um porta-filtro. A cabeça de amostragem, do fabricante TCR TECORA, com design simétrico, a fim de possibilitar a coleta de partículas com qualquer direção de vento, vertical, área da seção transversal para entrada de amostra com área de $12,13 \mathrm{~cm}^{2}$. A unidade de aspiração de amostra contava com um sistema de medição da vazão e um sistema de controle para atuação sobre a bomba, proporcionando um controle de vazão da amostra de $2 \%$, considerando-se a vazão de amostragem de $23,8 \mathrm{~L}$. $\mathrm{min}^{-1}$. A massa de partículas foi obtida por meio de gravimetria dos filtros em balança de precisão de 0,01 mg, pré e pós-amostragem. Os filtros foram submetidos à secagem em dessecador por 48 horas e temperatura ambiente, critério de equilíbrio das pesagens de $0,02 \mathrm{mg}$, sendo adotado o peso médio de 2 pesagens. A concentração de PTS foi determinada pela relação entre a massa de partículas retida e o volume de ar amostrado em condição padrão.

Tendo em conta que os métodos avaliados são ambos gravimétricos, e as amostras em ambos os métodos são submetidas à secagem de forma análoga, cabe observação sobre os princípios da amostragem de aerossóis, especialmente sobre o aspecto inercial das partículas suspensas. Uma amostra de aerossol simplesmente reproduz uma condição aproximada da sua concentração no ar. Isso ocorre uma vez que a condição isocinética na amostragem não é possível, em razão da variabilidade das condições do vento. Fatores podem influenciar na distribuição e na concentração de PTS amostrada, tais como distribuição da massa/tamanho das partículas no 
ar, geometria do sistema de amostragem, velocidade do vento, relação entre a superfície de partículas e a massa de partículas, e turbulência do ar, inclusive causada pelo próprio equipamento (VEREIN DEUTSCHER INGENIEURE, 1999). Estudos demonstram que no ar em repouso a eficiência de amostragem de partículas até $15 \mu \mathrm{m}$ resulta entre 96 e 100\%, e que há maior influência sobre a eficiência de amostragem de partículas de diâmetros maiores em decorrência de diferenças entre a velocidade de amostragem e a velocidade do ar ambiente (FUCHS, 1964). Resultados de PTS de um sistema de APV foram comparados aos de um sistema de supergrande volume, em que se notou correlação entre os resultados de 94 a 99\% quando amostragens foram realizadas em condição de vento pouco intenso e fração de PM10 acima de $64 \%$. E verificou-se correlação de $71,4 \%$ quando amostragens foram realizadas em condição de vento intenso e fração PM10 de 53,6\% (VEREIN DEUTSCHER INGENIEURE, 2012b). Ou seja, partículas com maior inércia tendem a ter menor eficiência de amostragem.

Assim, o tamanho e a distribuição das partículas no ar são aspectos relevantes na amostragem e na determinação da sua concentração, e não são constantes na atmosfera. Estudos realizados sobre concentração total de partículas e de suas frações em cinco diferentes localidades dos Estados Unidos verificaram variação de 50 a $90 \%$ da fração PM10 no PTS, dependendo das condições dos locais e das condições de amostragem (LUNDGREN; HAUSKNECHT; BURTON, 1984). Estudos realizados entre abril e dezembro de 2011, em Al Samha, na península Arábica, verificaram relação média PM10/PTS de 61\%, PM2.5/PTS de 31\% e PM2.5/ PM10 de 47\% (AL-JALLAD; RODRIGUES; AL-THANI, 2017). Em janeiro, fevereiro, março e junho de 2007, na cidade de Shijiazhuang, na China, foram realizados trabalhos que revelaram relações entre particulado inalável e particulado total de $65,8,79,82,7$ e $74,8 \%$, respectivamente (DUAN et al., 2015).

Quanto às fontes das partículas, estas podem ser decorrentes de fenômenos naturais ou antropogênicos.
O PM2.5 é originado, principalmente, por fontes de combustão. O PM10 é principalmente produzido por processos mecânicos, como atividades de construção e suspensão do pó em rodovias pelo tráfego e pelo vento. Partículas na faixa de $30 \mu \mathrm{m}$ tendem a sedimentar e a ser ressuspensas por ação antropogênica ou por ocasião de ventos. Estudos realizados na China apontam que a combustão do carvão, emissões veiculares e fontes industriais são os principais contribuintes do PM2.5 (PUI; CHEN; ZUO, 2014). Avaliações conduzidas em Taiwan concluíram que emissões de PM2.5 na região foram originadas da combustão do carvão e do óleo, das emissões veiculares e do processamento secundário do alumínio (HSU et al., 2016). Estudos realizados na Espanha apontam a correlação direta entre o PM10 e o tráfego viário, e em áreas urbanas afetadas por tráfego (AMATO et al., 2014). Partículas decorrentes do tráfego de veículos podem apresentar tamanhos consideravelmente diferentes, visto que são emitidas pela exaustão do motor, pela abrasão de pneus, da superfície da pista, dos freios, ou seja, têm origens diferentes (PANT; HARRISON, 2013). Operações e atividades com materiais na forma de pó ou granulada também podem ser geradores de emissões de partículas. Avaliações da concentração PTS no entorno de indústria de produção de cimento identificaram que as partículas eram originadas principalmente pelo tráfego em vias não pavimentadas e a partir das pilhas de estoque de matérias-primas, carregadas pela ação de ventos (ABRIL et al., 2015). Estudos em região portuária que movimenta materiais na forma de pó, incluindo fertilizantes, observaram relação direta entre a concentração de partículas no ar e a intensidade do vento, e que a ressuspensão dos materiais movimentados é uma fonte importante de partículas (ALMEIDA et al., 2014). Partículas com diâmetro superior a $30 \mu \mathrm{m}$ tendem a não permanecerem distribuídas uniformemente no ar em razão de sua propensão à sedimentação, e tendem a ser suspensas pela ação antropogênica ou de ventos (VEREIN DEUTSCHER INGENIEURE, 1999).

\section{OBJETIVOS}

Os objetivos do trabalho foram:

- Avaliar a concentração de PTS no ar utilizando o método de AGV, referência para o monitoramento de PTS em vários países, e utilizando o método de APV apresentado;

- Determinar faixa de equivalência de resultados entre o método de APV apresentado e o método de grande volume. 


\section{MATERIAIS E MÉTODOS}

O amostrador de grande volume utilizado era conforme metodologia definida por ABNT, do fabricante Energética, modelo Hivol PTS, número de série HPV 1070, sem sistema de controle automático de vazão, cuja faixa de operação especificada é de 1,1 e $1,7 \mathrm{~m}^{3} \cdot \mathrm{min}^{-1}$. O amostrador de pequeno volume utilizado era do fabricante TCR Tecora, modelo Skypost PM, número de série 844497 , com troca automática de filtros, com capacidade de $6 \mathrm{~m}^{3} \cdot \mathrm{h}^{-1}$, acoplado a uma cabeça de amostragem do fabricante TCR TECORA, referência AA99-010-0015SP. A vazão de amostragem aplicada no amostrador de pequeno volume foi de $23,8 \mathrm{~L}$. $\mathrm{min}^{-1}$, calculada a fim de se proporcionar a mesma velocidade de aspiração de amostra esperada para o amostrador de grande volume, de $32,7 \mathrm{~cm} . \mathrm{s}^{-1}$, visando à homogeneidade na coleta da amostra para ambos os equipamentos utilizados. As características principais dos métodos de determinação de PTS considerados são apresentadas na Figura 1.

Preliminarmente à amostragem em cada local, o amostrador de grande volume foi calibrado utilizando-se copo calibrador com manômetro, barômetro e termômetro. Foi efetuado ajuste aproximado da vazão a partir do ajuste do variador de tensão do motor. Entretanto, esse ajuste é aproximado, pois o equipamento não permite a verificação da vazão com precisão, somente o cálculo aproximado a partir da leitura da carta gráfica, considerando-se pressão atmosférica, temperatura do ar e coeficientes da calibração a2 e b2. Os filtros utilizados, brancos e amostrados, foram secos em umidade inferior a $50 \%$ por pelo menos 24 horas, até seu equilíbrio, e pesados com precisão de 0,1 mg. O amostrador de pequeno volume também foi preliminarmente calibrado em cada local amostrado utilizando-se um calibrador padrão de vazão digital, e os filtros brancos e amostrados foram submetidos à secagem em ambiente com umidade inferior a $50 \%$, até estabilização, e pesados com precisão de 0,01 mg. Os equipamentos foram instalados distando entre si aproximadamente $3 \mathrm{~m}$, em área distante de obstáculos em pelo menos $12 \mathrm{~m}$.

Foram realizadas amostragens em paralelo com os sistemas de grande e de pequeno volume de outubro de 2011 a janeiro de 2012, nas localidades de Curitiba $\left(25^{\circ} 26^{\prime} 48,70^{\prime \prime} \mathrm{S}, 49^{\circ} 21^{\prime} 31,88^{\prime \prime} \mathrm{O}\right)$, Sengés $\left(24^{\circ} 06^{\prime} 57,59^{\prime \prime} \mathrm{S}, 49^{\circ} 28^{\prime} 21,60^{\prime \prime} \mathrm{O}\right)$ e Paranaguá $\left(25^{\circ} 32^{\prime} 04,54^{\prime \prime} \mathrm{S}, 48^{\circ} 31^{\prime} 21,41^{\prime \prime} \mathrm{O}\right)$, no estado do Paraná, Brasil. O local de amostragem denominado $A$, no município de Curitiba, é área predominantemente urbana, com vias pavimentadas e condições de tráfego de automóveis moderado na maior parte do dia, apresenta tráfego intenso somente em alguns horários específicos, e não apresenta atividades industriais nas proximidades. Considerando as fontes de combustão veicular, havia expectativa de se obter resultados de concentração de PTS baixo a moderado, e de que a distribuição de partículas fosse predominantemente de partículas finas. O local denominado B, no município de Sengés, apresentava pouco trânsito de veículos e com presença de indústria papeleira no entorno, a população relatava sobre inconvenientes decorrentes de partículas em suspensão no ar, portanto havia expectativa de se obter resultados de concentração de PTS moderados. O local denominado $C$, no município de Paranaguá, tem atividade industrial de armazenamento e distribuição de fertilizantes e apresenta grande quantidade de material sólido em pó sobre o piso, decorrente da queda dos próprios produtos durante o transporte em caminhões. O material transportado tem aspecto visivelmente grosso e visualmente se verifica a ressuspensão do pó por ação de ventos e nos períodos de tráfego mais intenso. Determinações anteriores de PTS decorrentes do processo de licenciamento ambiental de empreendimento no local apresentaram violação do padrão diário de PTS e deram evidências de ser originário do processo de ressuspensão do próprio material existente sobre o solo. Assim, tinha-se a expectativa de encontrar partículas em suspensão com diâmetro elevado e de se obter resultados de concentração de PTS elevada.

\section{ANÁLISE ESTATÍSTICA}

A análise estatística dos resultados da concentração de PTS foi efetuada de quatro formas distintas. Foi efetuada a avaliação do grau de relação entre os resultados gerados com os métodos por meio da avaliação do coeficiente de correlação linear, em que resultados superiores a 0,9 caracterizam forte correlação positiva. 
Gravimetria de filtros (pré e pós-amostragem)

Amostragem de grande volume ABNT NBR 9.547/97

- Filtro retangular: $20,3 \times 25,4 \mathrm{~cm}$

- Balança de precisão: 0,1 mg

- Condicionamento: mínimo 24 h a 50\% UR

- Controle de pesagem: até equilíbrio
Amostragem de pequeno volume

- Filtro circular: diâmetro 47 mm

- Balança de precisão: 0,01 mg

- Condicionamento: mínimo 24 h a 50\% UR

- Controle de pesagem: até equilíbrio em 0,02 mg

\section{Calibração do amostrador}

\section{Amostragem de grande volume ABNT NBR 9.547/97}

- Aplicação de copo de calibração, placas de resistência de fluxo ou resistência variável, barômetro e termômetro

- Registro de deflexão em carta gráfica e cálculo

- Cálculos de coeficientes de calibração e R

\section{Amostragem de pequeno volume}

- Aplicação padrão de calibração digital para a vazão 23,8 L. min $^{-1}$ e correção sobre valor lido pelo equipamento, se necessário

\section{Amostragem}

\section{Amostragem de grande volume ABNT NBR 9.547/97}

- Vazão de amostragem (cond. padrão de gás):

$$
1,1 \text { a } 1,7 \mathrm{~m}^{3} \cdot \mathrm{min}^{-1}
$$

- Seção de adução de amostra: retangular $765 \mathrm{~cm}^{2}$

- Vazão ajustada a partir da calibração, porém não controlada ao longo da amostragem

$$
\text { - Tempo: } 24+/-1 \mathrm{~h}
$$

\section{Amostragem de pequeno volume}

- Vazão de amostragem (cond. padrão de gás): 23,8 L. $\min ^{-1}(+/-3 \%)$

- Seção de adução de amostra: circular 12,13 cm²

- Vazão controlada em condição padrão de gás

- Tempo: $24+/-1 \mathrm{~h}$

\section{Obtenção de resultados}

\section{Amostragem de grande volume ABNT NBR 9.547/97}

- Leitura de cartas gráficas, obtenção de médias de temperatura e pressão atmosférica, cálculos de vazões, volumes e concentração de partículas

\section{Amostragem de pequeno volume}

- Download de registros eletrônicos e cálculo de concentração de partículas

ABNT: Associação Brasileira de Normas Técnicas; NBR: Norma Brasileira; UR: umidade residual; cond.: condição.

Figura 1 - Características principais dos métodos de determinação de partículas totais em suspensão aplicados. 
A segunda forma aplicou o índice z para a avaliação dos resultados, com limites de aceitação dentro do intervalo entre $+2 z$ e $-2 z$ (THOMPSON; ELLISON; WOOD, 2006). O desvio padrão atribuído ao método de referência foi de $10 \mu \mathrm{g} \cdot \mathrm{m}^{-3}$ para resultados de concentração na faixa de concentração até $100 \mu \mathrm{g} \cdot \mathrm{m}^{-3}$, conforme Ente Nazionale Italiano di Unificazione (2001), uma vez que incertezas de resultados para baixas concentrações são decorrentes dos procedimentos de manuseio e gravimétrico. Já o desvio padrão adotado para concentrações acima de $100 \mu \mathrm{g} . \mathrm{m}^{-3}$ foi de 3,7\% do valor designado, que é o desvio padrão relativo estabelecido conforme a ABNT (1997), considerando intervalo de confiança de $95 \%$ (IC95\%).

A terceira forma de avaliação aplicou um teste de hipóteses tipo t para médias dos resultados de concentração das amostras obtidas com cada método.
Os resultados foram também avaliados por critérios específicos de linearidade e de desvio bilateral, com base em conceitos e critérios estabelecidos em ECTC (2010) e Ente Nazionale Italiano di Unificazione (2001). Inicialmente, avaliou-se a linearidade e, uma vez atendido este critério, avaliou-se o desvio bilateral. A linearidade é considerada aceitável caso o coeficiente angular (b) subtraído de um, em módulo, seja inferior a duas vezes a incerteza padrão do coeficiente angular $u(b)$, e se o coeficiente linear (a) seja muito próximo de zero ou no máximo duas vezes a incerteza padrão do coeficiente linear $u(a)$. $O$ desvio bilateral admissível foi de $10 \mu \mathrm{g} \cdot \mathrm{m}^{-3}$ para resultados de concentração na faixa de concentração até $100 \mu \mathrm{g} \cdot \mathrm{m}^{-3}$. Para resultados de concentração superiores a $100 \mu \mathrm{g} \cdot \mathrm{m}^{-3}$ o desvio bilateral admissível foi de $3,7 \%$, utilizando assim a incerteza dos resultados do método de referência conforme a ABNT (1997), em IC95\%.

\section{RESULTADOS E DISCUSSÃO}

As amostragens realizadas nos três locais definidos, A, $B$ e $C$, foram realizadas em condições de temperatura ambiente média entre 12,8 e $27,7^{\circ} \mathrm{C}$, com temperatura mínima registrada de $9^{\circ} \mathrm{C}$, temperatura máxima registrada de $37,4^{\circ} \mathrm{C}$, e em condições de pressão atmosférica média entre 90,69 e 101,47 KPa. Os resultados obtidos totalizaram 46 pares de dados de concentração de PTS, sendo dois pares de dados descartados por apresentarem resultados de concentração de PTS acima do valor máximo atribuído ao método de referência, $750 \mu \mathrm{g} \cdot \mathrm{m}^{-3}$.

Foram considerados 44 pares de resultado de PTS (Tabela 1). A concentração média de PTS e o desvio padrão verificados com o método AGV foram de 88,13 e $105,64 \mu \mathrm{g} . \mathrm{m}^{-3}$, superiores aos resultados obtidos com o método APV, de 71,28 e $68,63 \mu \mathrm{g} \cdot \mathrm{m}^{-3}$. A concentração de PTS mínima verificada com ambos os métodos foi semelhante $-13,39 \mu \mathrm{g} \cdot \mathrm{m}^{-3}$ para o método de referência e $15,25 \mu \mathrm{g} \cdot \mathrm{m}^{-3}$ para o método do APV. Entretanto, as concentrações máximas verificadas para os métodos foram mais discrepantes entre si, de 480,37 $\mu \mathrm{g} \cdot \mathrm{m}^{-3}$ para o método AGV e de $298,81 \mu \mathrm{g} \cdot \mathrm{m}^{-3}$ para o método APV. Dos 44 pares de dados considerados, 37 apresentaram resultados de concentração de partículas totais em suspensão de até $100 \mu \mathrm{g} \cdot \mathrm{m}^{-3}$. Os pares de dados 18 , $38,39,41,42$, 43 e 44 apresentaram maior amplitude (Figura 2). Os pares de dados de 38 a 44 foram obtidos no local C e o par 18, no local B.
A média da relação de velocidade de aspiração de amostra entre o método APV e o método AGV foi de $0,98,1,01$ e 0,95 nos locais A B e C, respectivamente. $E$ a média da relação de concentração entre os métodos APV e AGV foi de $0,87,1,00$ e 0,76 nos locais A B e $C$, respectivamente.

A correlação entre os resultados dos métodos foi de $0,95,0,97,0,91$ e 0,89 para a série integral, pares de dados do local A, do local B e do local C, respectivamente, demostrando forte correlação positiva.

$\mathrm{Na}$ avaliação estatística dos resultados de concentração de PTS utilizando o índice z (THOMPSON; ELLISON; WOOD, 2006), verifica-se que dos 44 pares de resultados de concentração de PTS ocorreram 6 com índice $z$ fora dos limites. Desses resultados, cinco foram provenientes de amostras obtidas na localidade $C$ e um na localidade B (Tabela 1).

A avaliação estatística a partir do teste de hipóteses tipo $\mathrm{t}$ foi aplicada para médias dos resultados de concentração das amostras obtidas com cada método (Tabela 2). $\mathrm{O} t$ valor foi inferior ao $t$ crítico para as amostras referentes aos locais A e B, entretanto foi superior para as amostras do local $\mathrm{C}$.

A avaliação estatística a partir da avaliação da linearidade para a série integral demonstrou que o coeficiente linear (a) 
Tabela 1 - Resultados de concentração (Conc.) de partículas totais em suspensão (PTS) e relação para os métodos amostragem de grande volume (AGV) e amostragem de pequeno volume (APV), velocidade de aspiração de amostra e relação para os métodos amostragem de grande volume e amostragem de pequeno volume, $e$ índice $z$.

\begin{tabular}{|c|c|c|c|c|c|c|c|c|c|c|}
\hline $\begin{array}{l}\text { Par de } \\
\text { dados }\end{array}$ & Localidade & $\begin{array}{c}\text { Vazão } \\
\text { média } \\
\text { AGV } \\
\text { (L. } \text { min }^{-1} \text { ) }\end{array}$ & $\begin{array}{c}\text { Vazão } \\
\text { média } \\
\text { APV } \\
\text { (L.min'-1) }\end{array}$ & $\begin{array}{l}\text { Velocidade } \\
\text { média AGV } \\
\left(\mathrm{cm} \cdot \mathrm{s}^{-1}\right)\end{array}$ & $\begin{array}{l}\text { Velocidade } \\
\text { média APV } \\
\left(\mathbf{c m} \cdot \mathrm{s}^{-1}\right)\end{array}$ & $\begin{array}{c}\text { Relação } \\
\text { velocidade } \\
\text { média APV/ } \\
\text { AGV }\end{array}$ & $\begin{array}{l}\text { Conc. de } \\
\text { PTS APV } \\
\left(\mu g \cdot m^{-3}\right)\end{array}$ & $\begin{array}{c}\text { Conc. de } \\
\text { PTS AGV } \\
\left(\mu g \cdot m^{-3}\right)\end{array}$ & $\begin{array}{c}\text { Îndice } \\
\text { Z }\end{array}$ & $\stackrel{\sigma_{p}}{\text { atribuido }}$ \\
\hline 1 & A & 1656 & 23.7 & 36.08 & 32.55 & 0.90 & 13.39 & 15.25 & -0.2 & 10 \\
\hline 2 & A & 1656.6 & 23.7 & 36.09 & 32.55 & 0.90 & 13.82 & 19.64 & -0.6 & 10 \\
\hline 3 & B & 1358.7 & 23.5 & 29.60 & 32.28 & 1.09 & 17.39 & 21.39 & -0.4 & 10 \\
\hline 4 & A & 1509.5 & 23.7 & 32.89 & 32.55 & 0.99 & 16.59 & 22.26 & -0.6 & 10 \\
\hline 5 & B & 1407.7 & 23.7 & 30.67 & 32.55 & 1.06 & 16.87 & 23.43 & -0.7 & 10 \\
\hline 6 & A & 1509.5 & 23.7 & 32.89 & 32.55 & 0.99 & 20.52 & 24.14 & -0.4 & 10 \\
\hline 7 & A & 1488.7 & 23.7 & 32.43 & 32.55 & 1.00 & 24.26 & 26.81 & -0.3 & 10 \\
\hline 8 & A & 1638.3 & 23.7 & 35.69 & 32.55 & 0.91 & 14.08 & 28.04 & -1.4 & 10 \\
\hline 9 & B & 1545.6 & 23.7 & 33.67 & 32.55 & 0.97 & 28.7 & 32.91 & -0.4 & 10 \\
\hline 10 & B & 1541.9 & 23.7 & 33.59 & 32.55 & 0.97 & 39.8 & 32.99 & 0.7 & 10 \\
\hline 11 & A & 1490.1 & 23.7 & 32.46 & 32.55 & 1.00 & 34.73 & 33 & 0.2 & 10 \\
\hline 12 & B & 1420.1 & 23.7 & 30.94 & 32.55 & 1.05 & 32.16 & 37.08 & -0.5 & 10 \\
\hline 13 & B & 1452.9 & 23.7 & 31.65 & 32.55 & 1.03 & 33.07 & 37.37 & -0.4 & 10 \\
\hline 14 & B & 1466.9 & 23.7 & 31.96 & 32.55 & 1.02 & 36.86 & 38.14 & -0.1 & 10 \\
\hline 15 & A & 1490.2 & 23.7 & 32.47 & 32.55 & 1.00 & 32.99 & 39.04 & -0.6 & 10 \\
\hline 16 & A & 1481.5 & 23.7 & 32.28 & 32.55 & 1.01 & 38.96 & 39.75 & -0.1 & 10 \\
\hline 17 & B & 1447.2 & 23.7 & 31.53 & 32.55 & 1.03 & 46.01 & 39.76 & 0.6 & 10 \\
\hline 18 & B & 1499.9 & 23.7 & 32.68 & 32.55 & 1.00 & 68.65 & 40.56 & 2.8 & 10 \\
\hline 19 & B & 1499.7 & 23.7 & 32.67 & 32.55 & 1.00 & 45.74 & 42.66 & 0.3 & 10 \\
\hline 20 & B & 1444.2 & 23.7 & 31.46 & 32.55 & 1.03 & 55.29 & 43.94 & 1.1 & 10 \\
\hline 21 & A & 1505.8 & 23.7 & 32.81 & 32.55 & 0.99 & 41.17 & 44.22 & -0.3 & 10 \\
\hline 22 & B & 1498.9 & 23.7 & 32.66 & 32.55 & 1.00 & 49.14 & 46.02 & 0.3 & 10 \\
\hline 23 & B & 1504.4 & 23.7 & 32.78 & 32.55 & 0.99 & 40.62 & 48.82 & -0.8 & 10 \\
\hline 24 & B & 1467.9 & 23.7 & 31.98 & 32.55 & 1.02 & 48.48 & 49.3 & -0.1 & 10 \\
\hline 25 & A & 1496.8 & 23.7 & 32.61 & 32.55 & 1.00 & 52.31 & 52.13 & 0.0 & 10 \\
\hline 26 & B & 1405 & 23.7 & 30.61 & 32.55 & 1.06 & 52 & 52.31 & 0.0 & 10 \\
\hline 27 & C & 1588 & 23.7 & 34.60 & 32.55 & 0.94 & 50.76 & 54.54 & -0.4 & 10 \\
\hline 28 & A & 1505.3 & 23.7 & 32.80 & 32.55 & 0.99 & 56.14 & 56.5 & 0.0 & 10 \\
\hline 29 & B & 1561.3 & 23.7 & 34.02 & 32.55 & 0.96 & 52.06 & 57.9 & -0.6 & 10 \\
\hline 30 & B & 1492 & 23.7 & 32.51 & 32.55 & 1.00 & 55.93 & 61.45 & -0.6 & 10 \\
\hline 31 & C & 1564.3 & 23.7 & 34.08 & 32.55 & 0.96 & 59.07 & 66.41 & -0.7 & 10 \\
\hline 32 & B & 1502.2 & 23.7 & 32.73 & 32.55 & 0.99 & 64.17 & 68.69 & -0.5 & 10 \\
\hline 33 & A & 1479.8 & 23.7 & 32.24 & 32.55 & 1.01 & 60.53 & 69.29 & -0.9 & 10 \\
\hline 34 & B & 1422 & 23.7 & 30.98 & 32.55 & 1.05 & 71.48 & 70.04 & 0.1 & 10 \\
\hline 35 & B & 1487 & 23.7 & 32.40 & 32.55 & 1.00 & 75.65 & 79.63 & -0.4 & 10 \\
\hline 36 & C & 1554.7 & 23.7 & 33.87 & 32.55 & 0.96 & 69.15 & 88.17 & -1.9 & 10 \\
\hline 37 & B & 1460.7 & 20.8 & 31.82 & 28.57 & 0.90 & 98.09 & 99.9 & -0.2 & 10 \\
\hline 38 & C & 1581.3 & 23.7 & 34.45 & 32.55 & 0.94 & 146.42 & 218.41 & -4.5 & 15.84 \\
\hline 39 & C & 1602.4 & 23.7 & 34.91 & 32.55 & 0.93 & 183.1 & 233.3 & -3.0 & 16.92 \\
\hline 40 & C & 1482.1 & 23.7 & 32.29 & 32.55 & 1.01 & 226.88 & 240.28 & -0.8 & 17.43 \\
\hline 41 & C & 1607.4 & 23.7 & 35.02 & 32.55 & 0.93 & 185.13 & 283.82 & -4.8 & 20.58 \\
\hline 42 & C & 1573.8 & 23.7 & 34.29 & 32.55 & 0.95 & 298.81 & 344.94 & -1.8 & 25.02 \\
\hline 43 & C & 1614.8 & 23.7 & 35.18 & 32.55 & 0.93 & 225.1 & 372.98 & -5.5 & 27.05 \\
\hline 44 & C & 1549.7 & 23.7 & 33.76 & 32.55 & 0.96 & 244.22 & 480.37 & -6.8 & 34.84 \\
\hline \multicolumn{2}{|l|}{ Média } & 1511.65 & 23.63 & 32.93 & 32.46 & 0.99 & 71.28 & 88.13 & - & - \\
\hline \multicolumn{2}{|c|}{ Mínimo } & 1358.70 & 20.80 & 29.60 & 28.57 & 0.90 & 13.39 & 15.25 & - & - \\
\hline \multirow{2}{*}{\multicolumn{2}{|c|}{ Máximo }} & 1656.60 & 23.70 & 36.09 & 32.55 & 1.09 & 298.81 & 480.37 & - & - \\
\hline & & 67.84 & 0.44 & 1.48 & 0.60 & 0.05 & 68.63 & 105.64 & - & - \\
\hline
\end{tabular}

AGV: amostragem de grande volume; APV: amostragem de pequeno volume; PTS: partículas totais em suspensão; $\sigma_{\mathrm{p}}$ : desvio padrão. 
foi inferior ao dobro da incerteza padrão do coeficiente linear u(a). Entretanto, o coeficiente angular (b) subtraído de um, em módulo, foi superior ao dobro da incerteza padrão do coeficiente angular $u(b)$, portanto a linearidade não foi adequada para a série integral de dados. Porém, ambos os critérios foram atendidos para a série de resultados de concentração de PTS obtidos nos locais A e B (Tabela 3).

Uma vez que os resultados referentes aos locais $A$ e $B$ atenderam aos critérios de linearidade, estes foram avaliados quanto à aceitação bilateral (Figura 3). Verifica-se que, entre os 37 pontos considerados, 33 atenderam ao critério de aceitação bilateral. Ainda, dos 34 resultados inferiores ao padrão médio anual de PTS, 31 atenderam ao critério de aceitação bilateral.

Quanto às origens e às características das partículas, nos locais A e B prevalecem partículas oriundas de combustão veicular e industrial, respectivamente, caracterizando particulado mais fino; fontes de combustão são

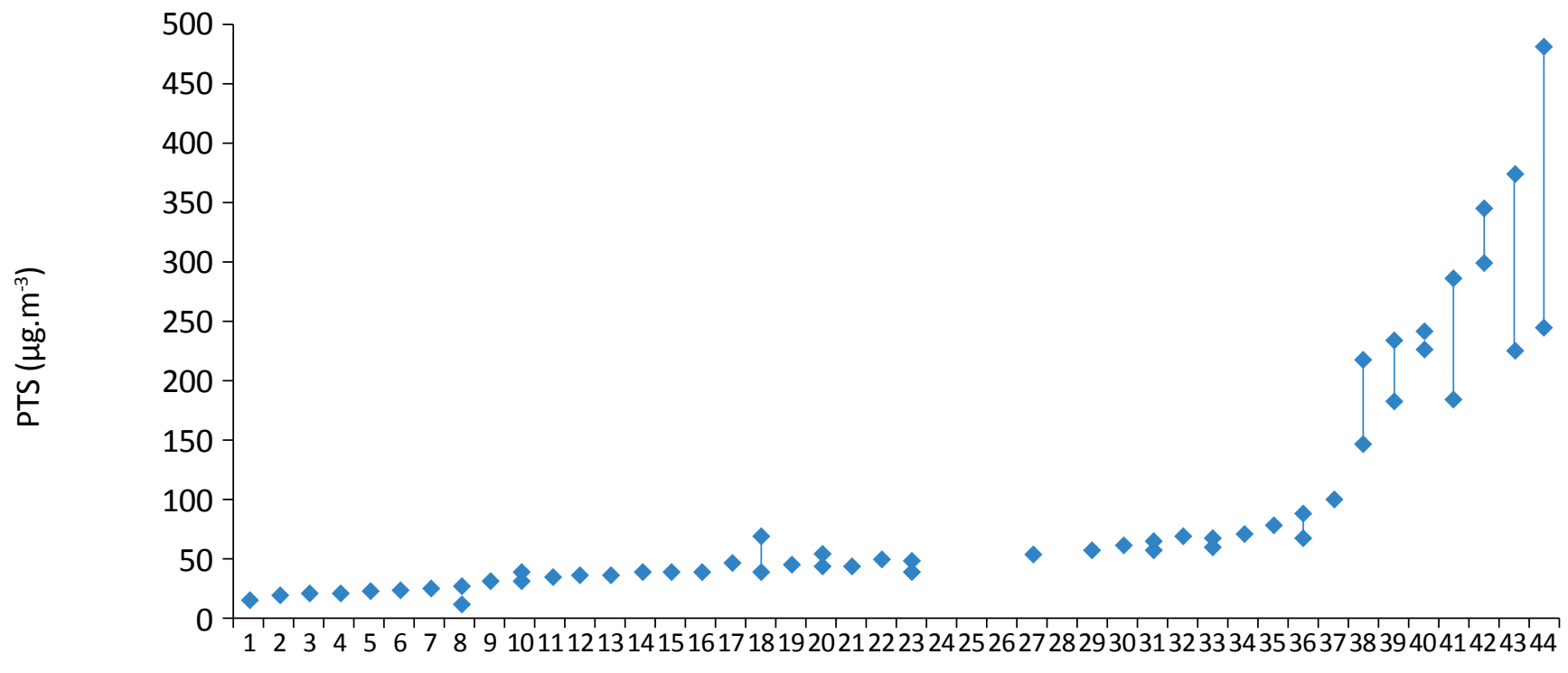

Amostra

Figura 2 - Amplitude dos resultados de concentração de partículas totais em suspensão (PTS) com os métodos da amostragem de grande volume (AGV) e da amostragem de pequeno volume (APV): série integral.

Tabela 2 - Teste de hipótese t de Student para as médias da concentração de partículas totais em suspensão verificada para as localidades A, B e C.

\begin{tabular}{|l|l|l|l|l|c|}
\hline \multicolumn{2}{|c|}{ Localidade A } & \multicolumn{3}{c|}{ Localidade B } & \multicolumn{2}{c|}{ Localidade C } \\
\hline$\alpha$ & 0,05 & $\alpha$ & 0,05 & $\alpha$ & 0,05 \\
\hline$\gamma$ & 12 & $\gamma$ & 20 & $\gamma$ & 9 \\
\hline $\mathrm{n}$ & 13 & $\mathrm{n}$ & 21 & $\mathrm{n}$ & 10 \\
\hline $\mathrm{d}$ médio & 4,18 & $\mathrm{~d}$ médio & 5,54 & $\mathrm{~d}$ médio & 69,46 \\
\hline$\sigma_{\mathrm{d}}$ & 3,90 & $\sigma_{\mathrm{d}}$ & 5,80 & $\sigma_{\mathrm{d}}$ & 74,22 \\
\hline$\Delta$ & 2,62 & $\Delta$ & 3,54 & $\Delta$ & 17,28 \\
\hline t valor & 1,443 & t valor & 1,585 & t valor & 2,223 \\
\hline t crítico & 1,78 & t crítico & 1,72 & t crítico & 1,83 \\
\hline
\end{tabular}

d médio: diâmetro médio; $\sigma_{\mathrm{d}}$ : variância do diâmetro. 
formadores do PM2.5. No local C, ocorrem especificamente atividades de descarga e armazenamento de fertilizantes. Assim, o particulado em suspensão no local $C$ tem grande contribuição a partir do fenômeno da ressuspensão do pó espalhado sobre o piso, durante o tráfego de caminhões, pela ação de ventos, bem como durante a descarga do material. Partículas com diâmetro superior a $30 \mu \mathrm{m}$ tendem a não permanecer distribuídas uniformemente no ar e tendem a ser suspensas pela ação antropogênica ou de ventos (VEREIN DEUTSCHER INGENIEURE, 1999). Ou seja, há presença significativa de partículas de maior diâmetro no local $C$, enquanto nos locais A e B prevalecem partículas de menor diâmetro.

A amostragem de PTS em locais com partículas de maior diâmetro e na ocorrência de ventos pode levar

Tabela 3 - Resultados dos cálculos para avaliação da linearidade para série integral e para resultados obtidos nos locais A e B.

\begin{tabular}{|l|c|c|}
\hline Resultados & Série integral & Locais A e B \\
\hline coeficiente linear - a & 15,116 & 2,652 \\
\hline incerteza padrão do coeficiente linear - u(a) & 7,973 & 6,193 \\
\hline duas vezes a incerteza padrão do coeficiente linear - 2u(a) & 15,946 & 12,383 \\
\hline coeficiente angular - b & 0,637 & 1,012 \\
\hline incerteza padrão do coeficiente angular - u(b) & 0,058 & 0,123 \\
\hline duas vezes a incerteza padrão do coeficiente angular - 2u(b) & 0,116 & 0,247 \\
\hline módulo do coeficiente angular subtraído de um - |b-1| & 0,363 & 0,012 \\
\hline
\end{tabular}

Concentração de PTS com AGV e APV - Locais A e B

Faixa $0-100 \mu \mathrm{g} \cdot \mathrm{m}^{3}$

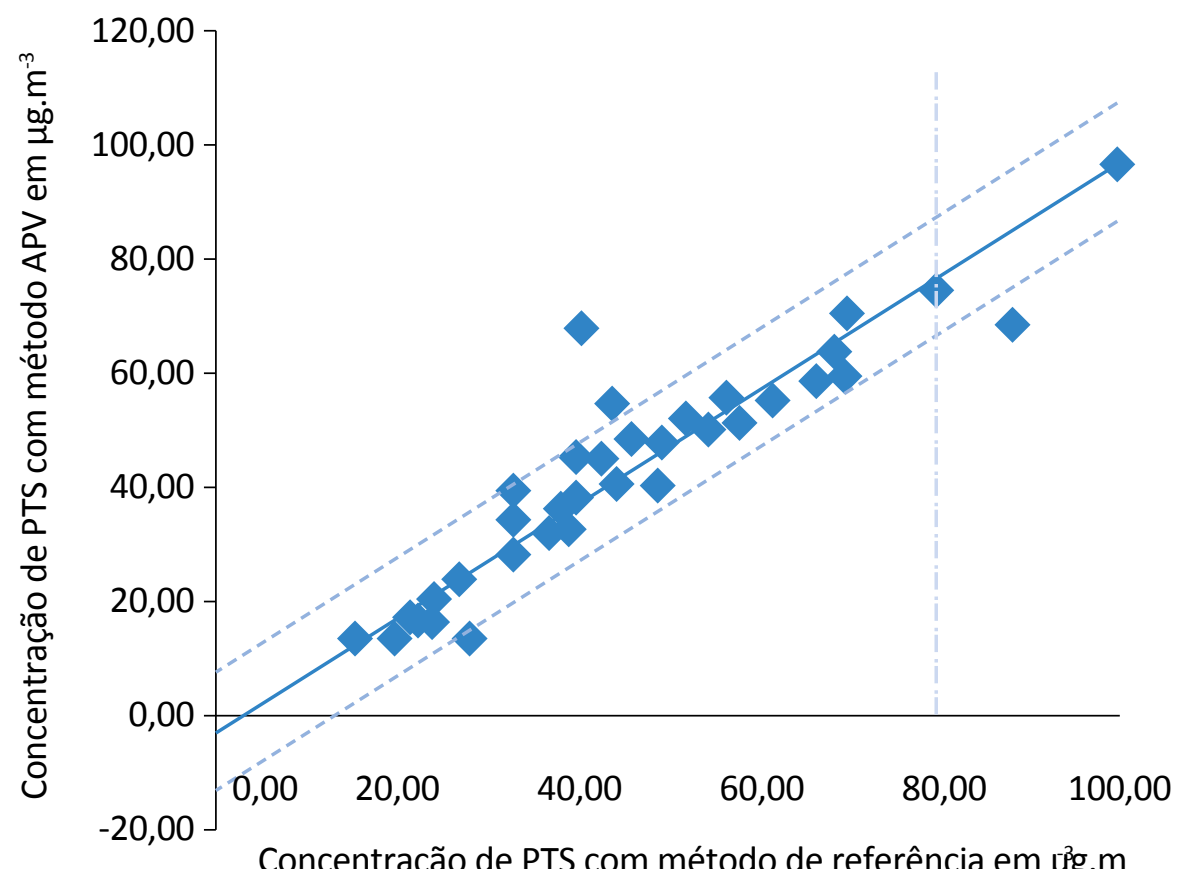

$y=1,0125 x-2,6521$

$\mathrm{R} 2=0,9290$

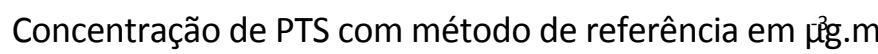

Figura 3 - Distribuição de pontos de concentração de partículas totais em suspensão (PTS) obtidos com os métodos da amostragem de grande volume (AGV) e da amostragem de pequeno volume (APV) - 0 a $100 \mu \mathrm{g} \cdot \mathrm{m}^{-3}$, locais A e B. 
a discrepâncias na amostragem utilizando-se sistemas de amostragem diferentes (VEREIN DEUTSCHER INGENIEURE, 2012b). Estudos sobre aerossóis demonstraram que partículas com diâmetro de $37 \mu \mathrm{m}$ amostradas em condição isocinética tiveram eficiência de coleta de $100 \%$, ou seja, não houve alteração na distribuição de partículas de $37 \mu \mathrm{m}$ amostradas. Na condição subcinética a $90 \%$, isto é, quando a velocidade de aspiração de amostra era $10 \%$ inferior à do ar passante, as partículas de $37 \mu \mathrm{m}$ tiveram eficiência de amostragem de aproximadamente $110 \%$, aumento de $10 \%$ na amostragem de partículas desse diâmetro. No mesmo estudo, verificou-se que partículas de $4 \mu \mathrm{m}$ não apresentaram alteração na eficiência de amostragem em condição isocinética, nem em condição 90\% subcinética (FUCHS, 1964). Considerando-se os resultados de concentração de PTS como métodos avaliados, no local C verificou-se a maior discrepância, a média da relação da concentração APV/AGV foi de 0,76 . Além disso, nesse local, constatou-se a maior discrepância quanto à relação de velocidade de aspiração de amostra entre os métodos. A média da relação da velocidade de aspiração APV/AGV foi de 0,95, sendo a velocidade média no AGV de $34,25 \mathrm{~cm} . \mathrm{s}^{-1}$. Cabe ressaltar que a velocidade média do ar na localidade foi de $111 \mathrm{~cm} \cdot \mathrm{s}^{-1}$ no período das amostragens, superior à velocidade de aspiração do ar nos amostradores. Logo, no local C, a menor velocidade de aspiração de amostra no APV em relação ao AGV, em condição de amostragem subcinética, e em atmosfera com partículas de maior diâmetro, levou à amostragem de partículas com distribuição de diâmetro inferior pelo APV, levando a resultados de concentração significativamente menores para esse método.

O método de referência admite uma faixa ampla de velocidade de aspiração de amostra, de 20 a $35 \mathrm{~cm} \cdot \mathrm{s}^{-1}$. Portanto, essa ampla faixa de velocidade confere a esse método um incremento à incerteza de seus resultados, sendo mais acentuada em atmosferas com partículas de maior diâmetro.

\section{CONCLUSÕES}

Os resultados demonstraram que o método do amostrador de pequeno volume apresentado é equivalente ao atual método de referência para determinação de PTS estabelecido na norma ABNT/NBR n ${ }^{\circ}$ 9.547/97, na faixa de 0 a 100 $\mu \mathrm{g} . \mathrm{m}^{-3} \mathrm{em}$ ambientes com partículas finas. $\mathrm{O}$ método do amostrador de pequeno volume apresentou resultados de PTS não equivalentes e inferiores aos obtidos com o método de referência em condições de atmosfera com partículas grosseiras originadas de ressuspensão de pó.

$O$ método de referência estabelecido em ABNT/NBR $n^{\circ}$ 9.547/97 define uma faixa de velocidade de aspiração de amostra ampla, de aproximadamente 20 a $35 \mathrm{~cm} \cdot \mathrm{s}^{-1}$.
Isso confere incremento à incerteza de seus resultados, principalmente em atmosferas com partículas de maior diâmetro. $O$ ajuste inicial da vazão de amostragem é de precisão restrita e apresenta limitação no seu controle durante a amostragem, o que contribui para o aumento da incerteza de seus resultados.

O método proposto possibilita ajuste inicial da vazão com precisão e controle automático ao longo da amostragem, portanto reduz incerteza dos resultados atribuída à amostragem. É uma alternativa mais flexível que permite amostragem de PM10 e PM2.5 pela troca da cabeça de amostragem.

\section{REFERÊNCIAS}

ABRIL, G. A.; DIEZ, S.; PIGNATA, M. L.; BRITCH, J. Particulate matter concentrations originating from industrial and urban sources: Validatlon of atmospheric dispersion modeling results. Atmospheric Pollution Research, v. 7, n. 1, 2015. http:// dx.doi.org/10.1016/j.apr.2015.08.009

AL-JALLAD, F. A.; RODRIGUES, C. C.; AL-THANI, H. A. Ambient Levels of TSP, PM10, PM2.5 and Particle Number Concentration in Al Samha, UAE. Journal of Environmental Protection, v. 8, n. 9, p. 1002-1017, 2017. https://doi. org/10.4236/jep.2017.89063 
ALMEIDA, S. M.; SILVA, A. V.; GARCIA, S. M.; PINHEIRO, T. Chemical profile of fugitive particulate emissions. Journal of Radioanalytical and Nuclear Chemistry, v. 300, n. 2, p. 653-661, 2014. https://doi.org/10.1007/s10967-014-2980-x

AMATO, F.; ALASTUEY, A.; DE LA ROSA, J.; CASTANEDO, G. Y.; DE LA CAMPA, A. M. S.; PANDOLFI, M.; LOZANO, A.; GONZÁLEZ, J. C.; QUEROL, X. Trends of road dust emissions contributions on ambient air particulate levels at rural, urban and industrial sites in southern Spain. Atmospheric Chemistry and Physics, v. 14, p. 3533-3544, 2014. https://doi.org/10.5194/acp-14-3533-2014

ASSOCIAÇÃO BRASILEIRA DE NORMAS TÉCNICAS (ABNT). NBR 9.547/97: Material Particulado em Suspensão no Ar Ambiente - Determinação da concentração total pelo método do amostrador de grande volume - método de ensaio. Rio de Janeiro: ABNT, 1997.

BLANCO-BECERRA, L. C.; MIRANDA-SOBERANIS, V.; HERNÁNDEZ-CADENA, L.; BARRAZZA-VILLAREAL, A.; JUNGER, W.; HURTADO-DÍAZ, M.; ROMIEU, I. R. Effect of particulate matterless than $10 \mu \mathrm{m}$ (PM10) on mortality in Bogota, Colombia: a time-series analysis, 1998-2006. Salud Publica de Mexico, v. 56, n. 4, p. 363-370, 2014.

BRASIL. Ministério do Meio Ambiente. Resolução do Conselho Nacional do Meio Ambiente (CONAMA) n 003 , de 1990. Diário Oficial da União, Brasília, Seção I, p. 15937-15939, 1990.

CESAR, A. C. G.; NASCIMENTO, L. F. C.; CARVALHO JR., J. A. Associação entre exposição ao material particulado e internações por doenças respiratórias em crianças. Revista de Saúde Pública, v. 47, n. 6, p. 1209-1212, 2013. http:// dx.doi.org/10.1590/S0034-8910.2013047004713

CESARONI, G.; BADALONI, C.; GARIAZZO, C.; STAFOGGIA, M.; SOZZI, R.; DAVOLI, M.; FORASTIERE, F. Long-term exposure to urban air pollution and mortality in a cohort of more than a million adults in Rome. Environmental Health Perspectives, v. 121, n. 3, p. 324-331, 2013. https://doi.org/10.1289/ehp.1205862

COLÔMBIA. Ministerio de Salud. Decreto 2 de 1982 - Normas de Calidad del Aire y sus Metodos de Medicion. Bogotá: Ministerio de Salud, 1982.

DAUMAS, R. P.; MENDONÇA, G. A. S.; LEON, A. P. de. Poluição do ar e mortalidade em idosos no município do Rio de Janeiro. Cadernos de Saúde Pública, Rio de Janeiro, v. 20, n. 1, p.311-319, 2004. http://dx.doi.org/10.1590/S0102-311X2004000100049

DUAN, J.; CHEN, Y.; FANG, W.; SU, Z. Characteristics and relationship of PM, PM10, PM2.5 concentration in a polluted city in northern China. Procedia Engineering, v. 102, p. 1150-1155, 2015. https://doi.org/10.1016/j.proeng.2015.01.239

ENTE NAZIONALE ITALIANO DI UNIFICAZIONE. UNI EN 12341: Determinazione del Particolato in Sospenzione PM10. Roma: Ente Nazionale Italiano di Unificazione, 2001.

EUROPEAN COMMUNITY TECHNICAL COMMITTEE (ECTC). Guide to the demonstration of equivalence of air monitoring methods. European Community Technical Committee, 2010. Disponível em: <http://ec.europa.eu/environment/air/ quality/legislation/pdf/equivalence.pdf>. Acesso em: 9 fev. 2013.

FUCHS, N. A. The mechanics of aerosols. Londres: Pergamon Press, 1964.

HSU, C. Y.; CHIANG, H. C.; LIN, S. L.; CHEN, M. J.; LIN, T. Y.; CHEN, Y. C. Elemental characterization and source apportionment of PM10 and PM2.5 in the western coastal area of central Taiwan. Science of the Total Environment, v. 541, p. 1139-1150, 2016. https://doi.org/10.1016/j.scitotenv.2015.09.122

KESAVACHANDRAN, N. C.; KAMAL, R.; BIHARI, V.; PATHAK, M. K.; SINGH, A. Particulate matter in ambient air and its association with alterations in lung functions and respiratory health problems among outdoor exercisers in National Capital Region, India. Atmospheric Pollution Research, v. 6, n. 4, p. 618-625, 2015. https://doi.org/10.5094/APR.2015.070

$\mathrm{KIM}$, K. H.; KABIR, E.; KABIR, S. A review on the human health impact of airborne particulate matter. Environment International, v. 74, p. 136-143, 2015. https://doi.org/10.1016/j.envint.2014.10.005 
KUMAR, A.; ATTRI, A. K. Correlating respiratory disease incidences with corresponding trends in ambient particulate matter and relative humidity. Atmospheric Pollution Research, v. 7, n. 5, p. 858-864, 2016. https://doi.org/10.1016/j. apr.2016.05.005

LUNDGREN, A.; HAUSKNECHT, B. J.; BURTON, R. M. Large particle size distribution in five cities and the effect on a new ambient particulate matter standard. Aerosol Science and Technology, v. 3, n. 4, p. 467-473, 1984. https://doi. org/10.1080/02786828408959033

MÉXICO. Secretaria de Salud. Modificación a la norma oficial mexicana NOM-025-SSA1-1993, de 2005. Diário Oficial, Distrito Federal, 2005.

NARDOCCI, A. C.; FREITAS, C. U.; PONCE DE LEON, A. C. M.; JUNGER, W. L.; GOUVEIA, N. C. Poluição do ar e doenças respiratórias e cardiovasculares: estudo de séries temporais em Cubatão, São Paulo, Brasil. Cadernos de Saúde Pública, Rio de Janeiro, v. 29, n. 9, p. 1867-1876, 2013. http://dx.doi.org/10.1590/0102-311X00150012

PANT, P.; HARRISON, R. M. Estimation of the contribution of road traffic emissions to particulate matter concentrations from field measurements: A review. Atmospheric Environment, v. 77, p. 78-97, 2013. http://dx.doi.org/10.1016/j. atmosenv.2013.04.028

PUI, D. Y. H.; CHEN, S. C.; ZUO, Z. PM2.5 in China: Measurements, sources, visibility and health effects, and mitigation. Particuology, v. 13, n. 1, p. 1-26, 2014. http://dx.doi.org/10.1016/j.partic.2013.11.001

REINO UNIDO. Department of Environment, Food and Rural Affairs. Methods for monitoring particulate concentrations. Reino Unido: Department of Environment, Food and Rural Affairs, 2005. cap. 5. Disponível em: <http://archive.defra.gov. uk/environment/quality/air/airquality/publications/particulate-matter/documents/ch5.pdf>. Acesso em: 13 ago. 2011.

ROMÃO, R.; PEREIRA, L. A. A.; SALDIVA, P. H. N.; PINHEIRO, P. M.; BRAGA, A. L. F.; MARTINS, L. C. Relação entre baixo peso ao nascer e exposição ao material particulado inalável. Cadernos de Saúde Pública, Rio de Janeiro, v. 29, n. 6, p. 1101-1108, 2013. http://dx.doi.org/10.1590/S0102-311X2013000600007

THOMPSON, M.; ELLISON, S. L. R.; WOOD, R. The International harmonized Protocol for the proficiency testing of analytical chemistry laboratories. Pure Applied Chemistry, v. 78, n. 1, p. 145-196, 2006. http://dx.doi.org/10.1351/ pac200678010145

U.S. ENVIRONMENTAL PROTECTION AGENCY (USEPA). List of designated reference and Equivalent Methods. Estados Unidos: USEPA, 2011. Disponível em: <http://www.epa.gov/ttnamti1/files/ambient/criteria/reference-equivalentmethods-list.pdf>. Acesso em: 9 fev. 2013.

VEREIN DEUTSCHER INGENIEURE. VDI2463 Blatt 1: Messen von Partikeln, Gravimetrische Bestimmung der Massen Konzentration von partikeln in der Aussenluft, Grundlagen. Düsseldorf: Verein Deutscher Ingenieure, 1999.

. VDI2463 Blatt 8: Messen von Partikeln - Nicht fraktionierendes Probenahmesystem zur Erfassung von Schwebstaub in der Außenluft und Innenraumluft für Low-Volume-Sampler (LVS). Düsseldorf: Verein Deutscher Ingenieure, 2012b.

WORLD HEALTH ORGANIZATION (WHO). Air quality guidelines. Global update 2005. Particulate matter, ozone, nitrogen dioxide and sulfur dioxide, 2005. Disponível em: <http://www.euro.who.int/en/health-topics/environment-and-health/ air-quality/publications/pre2009/air-quality-guidelines.-global-update-2005.-particulate-matter,-ozone,-nitrogendioxide-and-sulfur-dioxide>. Acesso em: 10 fev. 2013. 